\title{
Institutional Factors Affecting Uptake Of Voluntary Medical Male Circumcision Among The Sexually Active Male Populations In Busia County
}

\author{
Dan Macho Okumu
}

$\mathrm{PhD}$

\author{
DOI: 10.29322/IJSRP.11.12.2021.p12044 \\ http://dx.doi.org/10.29322/IJSRP.11.12.2021.p12044
}

\begin{abstract}
The general goal of this investigation was to analyse the institutional components influence on take-up of VMMC in Busia County. Voluntary Medical Male Circumcision (VMMC) is the surgical removal of the foreskin by a trained health worker. This study utilized blended techniques configuration including both subjective and quantitative approaches. The study focused on male populace from 20 years and above. The population for this study was 276 respondents. The study connected fundamentally essential information using both quantitative and subjective information. Quantitative information was gathered by utilizing questionnaire while subjective information was gathered utilizing Focus Group Discussions and inside and out Interviews with Key Informants from the recorded associations managing HIV and AIDS and SBCC in Busia County. Statistical Product for Social Sciences (SPSS) was utilized for information analysis. From the study discoveries, it turned out that a substantial number of the young around there have the best possible data on what MC involves. Amid the Focus Group Discussions, the members could graphically clarify the procedure that they were taken through amid their visits to the VMMC destinations. There is a constructive and factually huge connection between institutional factors and take-up of Voluntary Medical Male Circumcision. The study concludes that Voluntary Medical Male Circumcision (VMMC) is a proven biomedical intervention that has the potential to save millions of lives and significant resources if it can be scaled up in high HIV prevalence regions where male circumcision is uncommon.
\end{abstract}

Index Terms- Uptake, Voluntary Medical Male Circumcision, sexually active and institutional factors

\section{BACKGROUND TO THE STUDY}

$I^{\mathrm{n}}$ nstitutions such as the family, clans, peer organizations, religious groups, cultural groups, and HIV and AIDS support groups have influence in the adoption of behavior and social practices such as the VMMC (NASCOP, 2014). Cultural barriers to male circumcision from the study done among noncircumcising communities, was mentioned by most participants as a significant cultural characteristic that distinguished the Luo and Samia are from other communities, and some expressed fear that introducing circumcision could cause loss of this cultural identity (NASCOP, 2014).
Because circumcision carries great cultural import in most societies, WHO/UNAIDS (2013) recommended that the sociocultural context of traditional male circumcision should inform how VMMC programming is promoted. The meanings and associations people attach to circumcision should be considered when designing circumcision programs, as these will act as the filter through which MMC promotion will be received (Nyaga, 2015). For instance, how will communities respond to VMMC promotion when circumcision, or the lack of it, is a mark of citizenship, religious or cultural affiliation or a sign of 'otherness' that signals exclusion, marginalization or oppression (Tobian, et al 2015). Additionally, it is important to understand the meanings attached to traditional forms of circumcision. Earlier studies found that some ethnic groups in which circumcision is not commonly practiced disapprove of circumcision, using derogatory terms for a circumcised man or a man with a congenitally shortened prepuce (Wouabe, 2013).

There is an expressed perception among most people that promoting VMMC would lead to a misconception that MC was some "magic bullet" against HIV, which could have an adverse effect on other preventive methods (Avert, 2017). Some of the females claim that many of these men who come out of circumcision will feel that the sex speed governor has been removed. Now that they are cut, their chances of being infected have reduced it is like the speed governor has been removed. Men will no longer want to use condoms (Avert, 2017). Another fear mentioned in several studies focusing on VMMC among traditionally non- circumcising communities is that $\mathrm{MC}$ promoted to reduce HIV, men who decide to remain uncircumcised would be discriminated against. The common argument is that the uncircumcised men would be labeled as risky or assumed as HIV infected. This issue brings up the perception that promoting MC in the community could discriminate against uncircumcised men (Bulled et al, 2015).

In Kenya $84 \%$ of men are circumcised but the level is lower among the non-circumcising ethnic groups (40\%). In some regions, there is discrimination, punishment, bullying and beatings incase a man is discovered not circumcised (Wouabe, 2013). In these regions, circumcision is usually behavioured in Augusts and therefore the desire to conform is always an important motivation in places where majority of male are circumcised and the uptake of circumcision is very high. Changing social norms or creating new social norms requires shifting (1) people's paradigms about 
what they perceive to be right or true, and (2) people's expectations regarding normative behaviors.

Social norms that are deeply rooted in one's beliefs are the most difficult to change. Various approaches can be used to shift social norms toward positive norms through interpersonal and community dialogue, social mobilization, and advocacy. For example, gender norms and social expectations of the roles that men play in reproductive health affects their attitudes and behaviors about HIV and pregnancy prevention, gender-based violence, and their participation in pregnancy, childbirth, newborn care and child care. Programs that address the social construction of gender roles through group or peer education, community outreach, mobilization, and mass media campaigns, and promote policy-level changes that support positive social norms, have been shown to have effects on changing norms.

In Yemen, the Safe Age of Marriage intervention used a community-based approach to change social norms and community attitudes regarding early marriage, girls' education, and the rights of girl children, including educating communities about the social and health consequences of child marriages, gaining support for keeping girls in school as an alternative to child marriage, and securing the support of religious leaders and stakeholders to support of increased age of marriage. Social norms are usually understood by measuring individual attitudes (positive or negative feelings regarding an idea or behavior) and beliefs. To date, the most commonly used surveys (e.g., Demographic and Health Survey (DHS) and Multi-Cluster Indicator Survey (MICS) do not capture social norm data. Efforts are underway to develop ways to measure social norms.

These also influence preference for Voluntary Medical Male Circumcision. Studies in USA have shown a strong association between preference for VMMC and high socioeconomic status, whereas low uptake of the services is associated with low socio economic status. In contrast, the Demographic and Health Surveys in sub-Saharan African countries show no consistent association with socioeconomic status. For example, in Tanzania, higher rates of circumcision were observed among men with higher levels of education, of higher socioeconomic status living in urban areas, whereas in Lesotho, circumcision was most common among men with no education, in the lowest wealth quartile and living in rural areas. Social - lifestyles of the respondents, health seeking behavior and support groups. Social organizations and institutions also have a role to play in social and behavior change. These include the family, schools, religious organizations, workplaces, peer and friend networks, as well as HIV and AIDS support groups and other interested organizations.

\section{Problem Statement}

According to (WHO, 2013), male circumcision is one of the oldest and most common surgical procedures worldwide. It is practiced for cultural, religious and social reasons, however there are none circumcising communities. A study done in Pune, India among un-circumcised men 18 years and above on the acceptability of MC showed that the fear of pain (Kabira et al, 2017), upto $71.3 \%$ of respondents, was the main obstacle for acceptability, followed by cultural barriers $(40.9 \%)$, fear of stigma $(29.5 \%)$, fear of medical complications (27.2\%). Approximately $30 \%$ of the world's males aged 15 years or older are circumcised. Of these, around two thirds are Muslims living mainly in Asia, the
Middle East and North Africa, $0.8 \%$ are Jewish, and $13 \%$ are nonMuslim and non- Jewish men living in the United States of America.

In nations with high HIV predominance and low commonness of male circumcision, for example, Zimbabwe (Nachmias et al, 2016), consequences of Randomized Control Trials (RCTs) done in South Africa and Uganda have exhibited that VMMC decreases HIV transmission by up to $60 \%$ (Kabira et al, 2017). It has been shown that circumcising $80 \%$ of men forestalled $45 \%$ of new HIV contaminations between the years 2011 and 2015 (Lanham et al, 2012). Nonetheless, it is important that VMMC offers complete security to HIV infection $(\mathrm{MoH}$, 2015).

Kenya has a generally high predominance of male circumcision. In the Kenya AIDS Indicator Survey (KAIS) of 2007,85 percent of men detailed that they were circumcised. Fifty two (52 percent) of the uncircumcised males live in Nyanza region with another group living in Rift Valley, Nairobi and Western Kenya. Altogether, 73 percent of the evaluated 1.4 million HIVtainted people in Kenya hail from the four locales. The most astounding HIV pervasiveness rates among uncircumcised males between 15-64 years are in Nairobi (20.2 percent), Nyanza (17.3\%), Rift Valley (7.0\%) and Western locale (6.8\%), (Mojola, 2017). These territories were chosen as need districts for execution of VMMC to accomplish 80 percent inclusion $(860,000$ circumcisions) by July 2013 to diminish HIV transmission in Kenya (MoH, 2015).

The Samia ethnic community in Kenya, like Nilotes; Luo, Turkana, Teso and Pokot do not practice traditional Male Circumcision. These communities have registered high HIV prevalence rates compared to those that practice traditional MC despite various radio, television and Non-governmental interventions on the uptake VMMC. WHO/UNAIDS has recommended $\mathrm{VMMC}$ as an additional strategy in fighting HIV/AIDS in these regions. Funyula in Busia County is therefore a priority region in Kenya (Kabira et al, 2017). It is projected that by achieving an $80 \%$ VMMC coverage among males 15 to 49 years old by 2025, then a significant number of new HIV infections will be averted (Mojola, 2017).

MPHS-Kenya. (2015) noted a causal connection among circumcision and the decrease in HIV frequency through the use of SBCC model. Among the Samia Funyula males the SBCC model is yet to be adopted. Most government institutions and the NGOs working among stake holders working among the Samia Funyula to encourage VMMC uptake have continued to use communication methods that hardly can change non-circumcising culture. This study seeks to fill this gap by determining the institutional factors that can be used to encourage on VMMC uptake.

\section{Research Objective}

To examine the institutional factors that affects the uptake of voluntary medical male circumcision among the sexually active male populations in Busia County.

\section{LITERATURE REVIEW}

A study by (Mattson et al., 2015) in Rwanda to determine the factors that motivate or demotivate men to circumcise or allow 
their sons do so made the following findings as prohibitory; men reported that they were too old to for circumcision, whereas willingness was associated with young age, marital status and knowledge that MC protects them against HIV. The study concluded that many younger people were willing to get circumcised (Mattson et al., 2015). The researchers admit that there is knowledge gap regarding the perception and willingness to undergo MC by older nontraditionally circumcising Rwandan men.

Tobian, et al (2015) conducted a study in Zimbabwe to examine adoption of VMMC; several interventions were identified that are likely to move men more quickly through this process of change. For instance, while at the pre-intention stage, the aim of behavior change interventions should be to increase men's exposure to VMMC messages through targeted messaging. Social pressure and encouragement to ascribe positive values to VMMC help move men further toward the decision to act, and addressing men's fears about the procedure may remove the final barrier. The study also demonstrated that even with critical danger of contracting HIV and AIDS, the act of preventive practices was still low. For instance, $80.9 \%$ of the respondents revealed that they didn't utilize a condom in their last sex. This was disregarding the learning of preventive estimation of condoms being at $89.1 \%$. In the young people's culture, different qualities are unquestionably more essential than requesting precautionary measures from sexual accomplices. For instance, to demand the utilization of condoms would disregard the essential estimation of relational trust (Wouabe, 2013). The study also proposes that youngsters are mindful of the estimation of condoms as a boundary to contraception than avert HIV transmission.

The above study is predictable with information from the KDHS reports of 2003 and 2008-09 which demonstrated that there had been a checked enhancement in learning of HIV anticipation strategies among young people aged 15-19 years. For example, $75 \%$ of teenagers realized that somebody could diminish the danger of getting the HIV infection by utilizing a condom each time one had sex. This learning of condom utilization expanded from $67 \%$ in 2003 to $75 \%$ in 2009 (MPHS-Kenya. (2015).

In their study (Avert, 2017) saw that HIV and AIDS programs concentrated on the person as an operator of progress and neglected the social, social and monetary conditions that may hinder the capacity to complete certain choices at individual dimension. They further say that human sexuality ought to be comprehended as a social development that should be investigated inside a more extensive setting in which it is rehearsed. A report by The Office of Disease Prevention and Health Promotion (2006), a United States based office on utilization of communication methodologies to advise people on VMMC found that the space of communication is a vital component in uptake of VMMC. These unique communication factors, person's introduction to VMMC, scan for, and utilization of personal data has been depicted as a success in uptake of VMMC.

In his study (Wouabe, 2013) points to a deficiency of learning among writers in an undeniably convoluted world. To connect this information gap, Khan found there is a need of an expansion in exchange and communication among columnists and the common society. To him, medical issues are at the foundation of the financial, social, and political issues of Africa. He further expresses that as writers and communicators utilize their methods and disposition to illuminate the general population, their significant assignments are to be pertinent and important to the worries of Africans like the VMMC issue.

IAS (2013) conferences in Zambia found out that 40 percent of men going for circumcision or not were associated with traditional HIV risk factors such as men with two or more sexual partners than monogamous men and community norms about being circumcised are changing markedly. It was also reported that the so-regarded as secondary benefits of social conformity, sexual attractiveness and feelings of being in control as a man were considerably more critical in making the decision to undergo the surgery than the expected perceived direct health benefits (International AIDS Society, 2013). Conversely, VMMC campaign message emphasize that male circumcision is an additional prevention method for men, but that it does not replace measures such as delay in the onset of sexual relations, avoidance of penetrative sex, reduction in the number of sexual partners, and correct and consistent use of male or female condoms (Wouabe, 2013). This is enough evidence that communicating partial protection remains challenge a big (Nyaga, 2015).

\section{RESEARCH METHODOLOGY}

To evaluate the social and behaviour change Communication factors influencing the take-up of VMMC among the generally non-circumcising in Busia County, blended techniques inquire about structure was used at the essential exact research level where the scientist gathered subjective and quantitative information specifically from the exploration members through surveys, meetings and center gathering exchanges at that point joined the information gathered in the study contemplate Creswell, (2014). By receiving the blended techniques inquire about plan, the scientist could comprehend why there was low take-up of VMMC in the district and this conquered the shortcoming and issues liable to emerge from single strategy look into studies. The use of both subjective and quantitative techniques in this investigation helped in getting affirmation of discoveries through assembly of alternate points of view that influence the take-up of voluntary medical male circumcision in generally non circumcising network in Busia County. Because of the mix, the investigation profited from the upside of test overview and measurable strategies (measurement, representativeness and attribution) and the benefits of the subjective and participatory methodologies.

\section{RESEARCH FINDINGS}

Institutions such as the family, clans, peer organizations, religious groups, cultural groups, and HIV and AIDS support groups have influence in the adoption of behavior and social practices such as the VMMC. The research study therefore designed questions surrounding the family, church, learning institutions among others to determine how they influenced uptake of VMMC among the traditionally non-circumcising. 
Table: Spouse Unsupportive of VMMC

\begin{tabular}{lll}
\hline & Frequency & Valid Percent \\
\hline Strongly Agree & 123 & 49.4 \\
Agree & 16 & 6.4 \\
Not Sure & 30 & 12.0 \\
Agree & 31 & 12.4 \\
Strongly Disagree & 49 & 19.7 \\
Total & $\mathbf{2 4 9}$ & $\mathbf{1 0 0 . 0}$ \\
\hline
\end{tabular}

A majority of the respondents at $49.4 \%$ strongly agreed that since their spouses didn't support male circumcision they wouldn.t undertake VMMC. With that information, lack of inclusion of women/partners in VMMC campaigns is bound to affect uptake of VMMC. Previous studies link uptake of male circumcision and spouses influence. According to (Kibira et al2017) men's motivation to seek circumcision is strongly related to their sexual partners where they could influence directly or indirectly by explicitly telling them to seek VMMC services or inexplicitly discussing circumcision benefits. An interview with a Program Officer expounded on effects of unsupportive spouse as outlined in the excerpt below;

We are working towards including working with men spouses so as to encourage older men to get involved in the VMMC programs. Women be it wives, girlfriends and mothers can be effective change agents thus it has been important as we push for male circumcision to work with them closely especially where men are reluctant because of their age or marital status. It is also important for women to understand that VMMC benefits not only the men but also the women in terms of preventing cervical cancer and enhanced sexual performance. We can attribute involvement of a good number of married men due to influence from their spouses. (Impact Research Org, Program Officer)

Table : Spouse Supports VMMC

\begin{tabular}{lll}
\hline & Frequency & Valid Percent \\
\hline Strongly Agree & 35 & 14.1 \\
Agree & 12 & 4.8 \\
Not Sure & 127 & 51.0 \\
Disagree & 23 & 9.2 \\
Strongly Disagree & 52 & 20.9 \\
Total & $\mathbf{2 4 9}$ & $\mathbf{1 0 0 . 0}$ \\
\hline
\end{tabular}

This publication is licensed under Creative Commons Attribution CC BY.
Only $14.1 \%$ of the respondents strongly agreed that their spouses supported male circumcision so they would undertake voluntary medical male circumcision, a majority of the respondents at $51 \%$ indicated that they were not sure if their spouse supported male circumcision which ultimately affects their decision on participating in VMMC. This uncertainty would affect uptake of VMMC thus campaign messages ought to target the women as well. According to (Kabira et al, 2017) African women are considered heath custodians in their communities; in this light, women therefore have an integral role in raising demand for VMMC given their influence on men. The VMMC communication campaigns will be more effective if women are factored in as well.

Table: Supports VMMC despite Family being unsupportive

\begin{tabular}{lll}
\hline & Frequency & Valid Percent \\
\hline Strongly Agree & 66 & 26.5 \\
Agree & 28 & 11.2 \\
Not Sure & 77 & 30.9 \\
Disagree & 29 & 11.6 \\
Strongly Disagree & 49 & 19.7 \\
Total & $\mathbf{2 4 9}$ & $\mathbf{1 0 0 . 0}$ \\
\hline
\end{tabular}

Only $37.7 \%$ of the respondents indicated that though their family didn't support male circumcision they would participate in VMMC, $30.9 \%$ were not sure if they would while $31.3 \%$ of the respondents disagreed. The influence of family in uptake of VMMC cannot be underestimated and as such campaign messages need to factor in secondary audiences as they are key in ensuring effectiveness of VMMC programs. A case study as outlined in (Nyaga, 2015) shows the power of family as an uncircumcised male refuses to participate in VMMC program because the family has cautioned him not to partake of it. The influence of other family members cannot be underestimated and messages need to factor that in.

Table: Church and VMMC

\begin{tabular}{lll}
\hline & Frequency & Valid Percent \\
\hline Strongly Agree & 45 & 18.1 \\
Agree & 26 & 10.4 \\
Not Sure & 66 & 26.5 \\
Disagree & 53 & 21.3 \\
Strongly Disagree & 59 & 23.7 \\
Total & $\mathbf{2 4 9}$ & $\mathbf{1 0 0 . 0}$ \\
\hline
\end{tabular}

28. $5 \%$ of the respondents agreed that since their church did not support male circumcision they wouldn.t participate in 
VMMC compared to $45 \%$ who didn't ascribe to this view, $26.5 \%$ were not sure. Every avenue that can push for uptake of VMMC needs to be included in designing and passing messages to encourage males to be part of the program. Religion has been reported as a barrier to seeking VMMC in many studies examining acceptability of male circumcision (Wouabe, 2013) it is therefore prudent to work with churches as they can be crucial in influencing uptake of circumcision especially amongst the traditionally non circumcising communities.

Table: Would Recommend VMMC to others

\begin{tabular}{lll}
\hline & Frequency & Valid Percent \\
\hline Strongly Agree & 110 & 44.2 \\
Agree & 12 & 4.8 \\
Not Sure & 61 & 24.5 \\
Disagree & 40 & 16.1 \\
Strongly Disagree & 26 & 10.4 \\
Total & $\mathbf{2 4 9}$ & $\mathbf{1 0 0 . 0}$ \\
\hline
\end{tabular}

A majority of respondents at $44.2 \%$ strongly agreed that having undergone male circumcision they would encourage my other family members and friends to undertake VMMC. The best drivers to increase VMMC uptake can only be those who have undergone the procedure as their testimonials can go a long way to positively influence those yet to be circumcised. Use of testimonials in VMMC campaign messages needs to be factored in as it is a persuasive way of encouraging others to be part of it. Use of men who have undergone VMMC and satisfied by the results are being utilized in other non-circumcising areas to motivate others which has seen its uptake go up (Avert, 2017). Thus it is a sure motivator to generate demand for VMMC in noncircumcising regions.

Table: Anti-VMMC because of Clan Disapproval

\begin{tabular}{lll}
\hline & Frequency & Valid Percent \\
\hline Strongly Agree & 91 & 36.5 \\
Agree & 13 & 5.2 \\
Not Sure & 76 & 30.5 \\
Disagree & 29 & 11.6 \\
Strongly Disagree & 40 & 16.1 \\
Total & $\mathbf{2 4 9}$ & $\mathbf{1 0 0 . 0}$ \\
\hline
\end{tabular}

A majority of the respondents at $41.7 \%$ were of opinion that since their clan disapproved male circumcision they wouldn.t therefore undertake VMMC, $30.5 \%$ were not sure while $27.7 \%$ disagreed. Without a traditional custom of circumcision many people may not see a compelling reason to undergo VMMC. Report by (Wouabe, 2013) indicates that to avoid conflicts with elders or family who don.t approve contravening traditions, men may shun VMMC programs. It is therefore crucial to consider working with secondary audiences such as clan elders when planning VMMC campaign messages.

Table: Undertook VMMC because of peer pressure in learning institutions

\begin{tabular}{lll}
\hline & Frequency & Valid Percent \\
\hline Strongly Agree & 62 & 24.9 \\
Agree & 16 & 6.4 \\
Not Sure & 31 & 12.4 \\
Disagree & 48 & 19.3 \\
Strongly Disagree & 92 & 36.9 \\
Total & $\mathbf{2 4 9}$ & $\mathbf{1 0 0 . 0}$ \\
\hline
\end{tabular}

Only $31.3 \%$ of the respondents indicated that they undertook VMMC because of peer pressure in the learning institutions. Social pressure has been recognized as a factor that could influence uptake of VMMC (Wouabe, 2013). This however, is applicable in younger males in learning institutions where they move in groups and are likely to influence one another. According to (Muhangi, 2010) peer pressure was a key determinant of the uptake of VMMC among young people mostly in the noncircumcising areas.

Table: Implementation of programs supporting VMMC at work places

\begin{tabular}{lll}
\hline & Frequency & Valid Percent \\
\hline Strongly Agree & 165 & 66.3 \\
Agree & 23 & 9.2 \\
Not Sure & 31 & 12.4 \\
Disagree & 14 & 5.6 \\
Strongly Disagree & 16 & 6.4 \\
Total & $\mathbf{2 4 9}$ & $\mathbf{1 0 0 . 0}$ \\
\hline
\end{tabular}

A majority of respondents at $66.3 \%$ strongly agreed that implementation of programs ensuring employers support employees who undergo circumcision and provide time off work would promote VMMC. Study by (Avert, 2017) points out that too much time away from work especially where man is the sole provider for the family as the most significant barrier to seeking VMMC services. There is need for VMMC campaigners to work with employers in areas where circumcision isn.t a priority to implement programs that support time away for men who undertake VMMC. 
Table: Correlations between institutional factors and VMMC

\begin{tabular}{llll}
\hline & & \multicolumn{1}{c}{$\begin{array}{c}\text { Institutional } \\
\text { VMMC factors }\end{array}$} \\
\hline VMMC & Pearson Correlation & 1 & $.203^{* *}$ \\
& Sig. (2-tailed) & & .001 \\
& $\mathrm{~N}$ & 249 & 249 \\
Institutional & Pearson Correlation & $.203^{* *}$ & 1 \\
factors & Sig. (2-tailed) & .001 & \\
& $\mathrm{~N}$ & 249 & 249 \\
**. Correlation is significant at the 0.01 level (2-tailed). & \\
\hline
\end{tabular}

The study further sought to describe the linear relationship between institutional factors and uptake of VMMC. The findings are shown in Table 4.36, which indicate that the Pearson correlation is $(r=0.203, P=0.001)$ which signifies a statistically significant association between institutional factors and uptake of VMMC since $\mathrm{P}<0.05$. It is true therefore to conclude if institutions influence VMMC uptake.

\section{CONCLUSION}

Like other studies conducted in African countries, the current study showed that adults were mostly knowledgeable about VMMC and knew the advantages of VMMC for health. VMMC prevalence in the area was higher than the national figure, while low uptake was associated with religion and education. Accomplishing the high national VMMC targets in Busia will be an important contributor to furthering the HIV decline in Busia. Guidelines and other practical tools have been developed to help programme managers and providers introduce or expand voluntary medical male circumcision (VMMC) services. They include guidance on conducting a situation analysis, establishing a VMMC programme, delivering services safely, efficiently, and effectively, addressing legal and ethical issues, managing the waste generated by VMMC services, and estimating the potential cost and impact of VMMC scale-up in Busia.

The governments of priority counties lead national efforts to implement voluntary medical male circumcision (VMMC) for HIV prevention, and most have developed VMMC policies and strategies. In recent years the emphasis has been on developing costed operational plans with specific targets for VMMC coverage, to guide the activities of Ministry of Health staff and their implementing partners.

Monitoring and evaluation (M\&E) of voluntary medical male circumcision programmes enables programme managers and independent evaluators to assess whether programmes are being implemented as intended, their reach, and their impact. The results can then be used to strengthen programmes.

\section{RECOMMENDATIONS}

The study recommends that the Ministry of Health and partners should increase the accessibility of VMMC through strong awareness and mobilization initiatives in the community. This goal should be achieved by creating more awareness about the safety and quality of services in government health facilities. In addition, providing health education in public and private institutions reinforcing that VMMC reduces female-to-male transmission risk of HIV and prevents STIs could lead to increased uptake of VMMC services in Busia. Further research is needed on uptake variables nationwide. This could involve those who have undergone $\mathrm{MC}$ and can share with others the advantages of the under taking. Institutions such as the family, clans, peer organizations, religious groups, cultural groups, and HIV and AIDS support groups should have influence in social practices such as the VMMC.

National programmes should conduct assessments of VMMC knowledge, attitudes, and practices and situation analyses to inform the design of strategies and services to expand access to voluntary medical male circumcision (VMMC). As scale-up progresses, monitoring and evaluation reports and the results of evaluation studies are providing recommendations for improving the safety, efficiency, and reach of VMMC programmes.

A number of suggestions were made in order to accelerate and maximize the impact of VMMC, including:

- Promoting VMMC as cost-effective in order to secure more funding from donors

- Allowing VMMC to be performed by nurses and other healthcare workers (task shifting)

- Prioritizing sub-populations (for example by age) in order to maximize a programme's impact and efficiency

- Using Geographic Information System (GIS) mapping to identify the areas where VMMC uptake is low

- Exploring the role of technologies in order to make circumcision more attractive to men.

\section{REFERENCES}

[1] Avert. (2017). Voluntary medical male circumcision for HIV prevention. Retrieved July 25, 2018, from www.avert.org: https://www.avert.org/professionals/hivprogramming/prevention/voluntary-medical-male-circumcision

[2] Bulled, N., \& Green, E. (2015). Making voluntary medical male circumcision a viable HIV prevention strategy in high-prevalence countries by engaging the traditional sector. Critical Public Health, 410-426.

[3] Creswell, J. W. (2014). Research Design: Qualitative, Quantitative and Mixed Methods Approaches 4th Ed. London: Sage Publications Ltd.

[4] Kabira, W. M., Gachukia, E. W., \& Matiangi, F. (2017). The Effect of Women.s Role on Health: The Paradox. International Journal of Gynecology and Obstetrics, 23-34.

[5] Lanham, M., L.Engle, K. L., Loolpapit, M., \& Oguma, I. O. (2012). Women.s Roles in Voluntary Medical Male Circumcision in Nyanza Province, Kenya. PLOS One.

[6] Mattson, C., Bailey, R., Muga, R., Poulussen, R., \& Onyango, T. (2015) Acceptability of male circumcision and predictors of circumcision preference among men and women in Nyanza Province, Kenya. AIDS Care, 182-194.

[7] MoH. (2015). National Voluntary Male Medical Circumcision Strategy 2014/15-2018/19. Nairobi: National AIDS and STI Control Program (NASCOP).

[8] Mojola, S. (2017). Fishing in dangerous waters: Ecology, Gender and Economy in HIV risk. Social Science and Medicine, 149-156. 
[9] MPHS-Kenya. (2015). Kenya National Health Strategy for Voluntary Medical Male Circumcision. Nairobi: Ministry of Public Health and Sanitation-Kenya.

[10] Muhangi, D. (2010). Factors that influence decisions to seek medical male circumcision services. USAID/JHU Associate Cooperative Agreement no. 617-A-00-07.0005-00.

[11] Nachmias, C. F., \& Nachmias, D. (2016). Research Methods in the Social Sciences (6th ed.). New York: Worth Publishers.

[12] NASCOP. (2014). Kenya AIDS Indicator Survey 2012: Final Report. Nairobi: National AIDS and STI Control Programme.

[13] Nyaga, E. M. (2015). Factors associated with uptake of voluntary medical male circumcision among men aged 18-50 years in kibera sub-county, nairobi county . Nairobi: Unpublished Thesis.
[14] Tobian, A. A., Adam, T., J. B., Kiggundu, V., Yazdi, Y., \& Njeuhmeli, E. (2015). Voluntary medical male circumcision in resource constrained settings. Macmillan Publishers Limited.

[15] World-Health-Organisation. (2013). Progress in scaling up voluntarymedical male circumcision for HIV prevention in East and Southern Africa JanuaryDecember 2012. Brazaville: World-Health-Organisation.

[16] Wouabe, E. D. (2013). Scoping Report on Interventions for Increasing the Demand for Voluntary Medical Male Circumcision. International Initiative for Impact Evaluation.

\section{AUTHORS}

First Author - Dan Macho Okumu, PhD 